\title{
Programmable Silicon Photonic Integrated Circuits
}

\author{
(Invited Paper) \\ Wim Bogaerts, Xiangfeng Chen, Mi Wang, Iman Zand, Hong Deng, Lukas Van Iseghem, \\ Antonio Ribeiro, Alejandro Diaz Tormo, Umar Khan \\ Ghent University - IMEC, Department of Information Technology, Photonics Research Group \\ Center of Nano -and Biophotonics, Ghent University, Ghent, Belgium \\ wim.bogaerts@ugent.be
}

\begin{abstract}
Programmable photonic circuits offer a new way to explore photonic chips: instead of hardwiring waveguide connections, one programs a mesh of tunable couplers. This requires electronics and software routines to govern the behavior of the chip, making it possible to rapidly develop new photonic applications.
\end{abstract}

\section{INTRODUCTION}

Photonic integrated circuits (PIC) are getting more complex as technology is improving. Especially with silicon photonics circuits, thousands of building blocks (couplers, phase shifters, photodetectors and even amplifiers) can be integrated onto a chip [1]. But today, most of these circuits are designed for a single application: they are application-specific PICs, or ASPICs. That means that a new chip needs to be designed for every new need, which slows down deployment in new applications [2].

What if PICs could follow the patterns of programmable electronics? Off-the-shelf available microcontrollers, digital signal processors (DSP) and field-programmable gate arrays (FPGA) can be configured to perform various tasks and make it possible to implement new functions in a matter of days, rather than months to design and fabricate a new chip.

The emerging field of programmable PICs tries to fill this space [3]. Rather than a specialized circuit of functional building blocks for a specific function, a programmable photonic circuit uses a mesh of waveguides with tunable couplers to define the connectivity on the chip at run-time in software through driver electronics. In this conference paper, we will explain the basic principles behind such generic programmable PICs, and where the technological needs differ from today's ASPICs.

\section{Programmable Waveguide Meshes}

At the heart of a programmable PIC is a mesh of interconnected waveguides, where the flow of light is controlled using tunable couplers and electro-optic phase shifters. A implementation is with a Mach-Zehnder interferometer with dual phase shifters, which can control the coupling ratio and phase delay independently [6]. It is not trivial to implement tunable couplers that cover a broad wavelength range with a full 0-100\% tuning range, but this can be solved by using additional circuit elements and active control [5].

Depending on the connection topology, we can identify two broad classes of meshes: forward-only and recirculating. In a forward-only mesh, light flows in one direction, and the couplers control the interference between different paths [6]. Such meshes are well suited for input-to-output switching, or for linear transformations between input and output modes, implementing matrix operations for artificial neural networks and quantum-optic circuits [7].

In recirculating meshes, the waveguides are arranged in loops, which are coupled along the edges. This way, light can be coupled back onto itself, forming delay lines and resonators. The delays and loops can form interferometric wavelength filters [4] to select specific optical wavelength bands, or process radio-frequency (RF) signals modulated on an optical carrier.

The reconfigurability of the mesh is only possible with many electrically controlled tunable couplers and phase shifters. These need to be compact and have a low optical loss, because the light passes through many actuators in series. In silicon photonics, the most common phase shifters are based on heaters, but these consume a lot of power, which does not scale well to larger circuits. Les mature alternatives include piezo-electric actuators, liquid crystals and MEMS [8].

\section{WHAT IS NEEDED FOR A PROGRAMMABLE PIC?}

While the waveguide mesh core is essential to reconfigure the PIC, additional elements are needed for a generic fully programmable PIC. First of all are inputs and outputs for signals: for optical signals, fiber interfaces are most straightforward (surface gratings or edge couplers). But it is also possible to use high-speed electro-optic modulators to translate RF to the optical domain, and (balanced) photodiodes to translate modulated optical signals back to the RF domain.

The programmable PIC needs driver and control electronics, to control the many the phase shifters and tunable couplers independently. This requires with many electrical channels which need to be connected to the chip. We have developed a

This work was funded by the European Union through Grants 780283 (MORPHIC) and 725555 (PhotonicSWARM) 


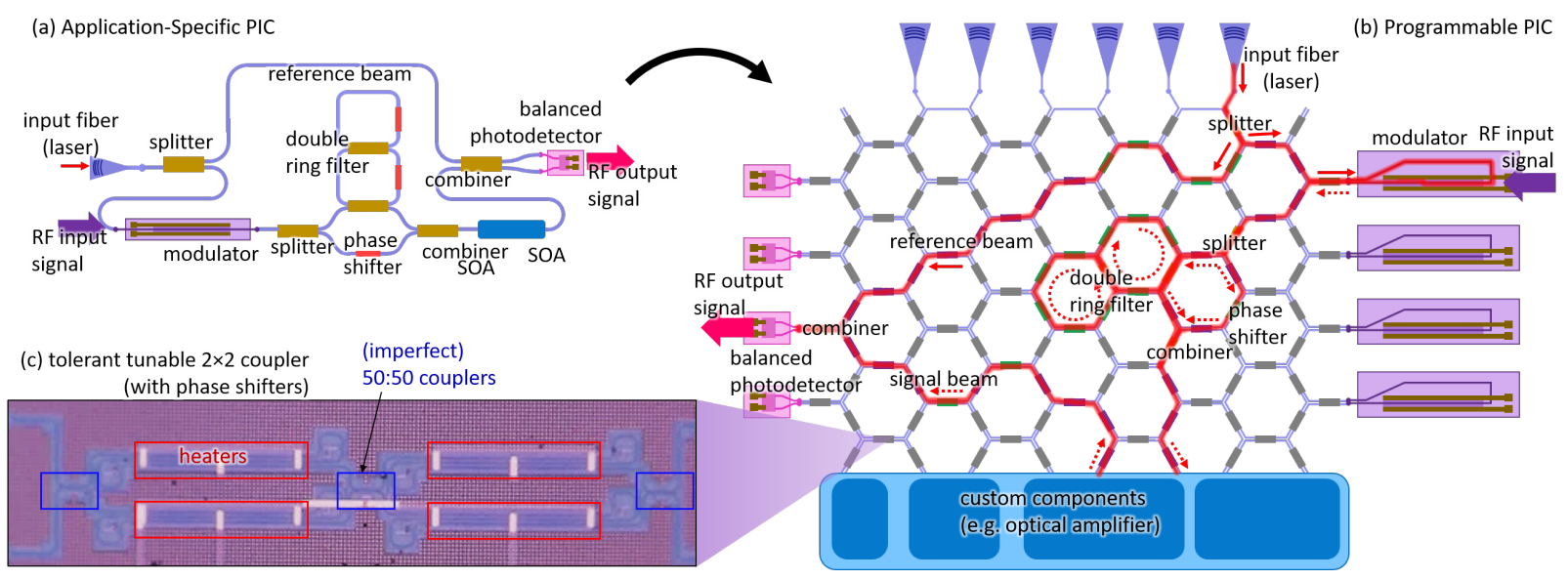

Fig. 1. Architecture of a programmable PIC with a hexagonal recirculating mesh [4]. (a) a design for a photonic circuit can be programmed into (b) the programmable PIC, which consists of a mesh of (c) tunable couplers with phase shifters [5]. Radio-frequency (RF) signals are modulated on an optical carrier, processed in the optical domain, and then converted back to an RF signal.

multiplexing scheme based on matrix addressing that dramatically increases the number of thermo-optic phase shifters that can be controlled simultaneously with a limited number of connection wires [9]. These drivers need to be controlled with feedback loops that use signals of monitor detectors that are integrated on the photonics chip [10].

\section{Programming a PIC}

On top of the photonics and electronics, different layers of software are needed to govern the behavior. Low-level control routines are needed to keep the circuit stable [10], while high-level programming routines help the user to configure the circuit to a desired behavior, such as routing light between input and output ports [11], [12].

Based on such algorithms, the tunable couplers and phase shifters along the path of the light can be configured. This should result in a near-perfect behavior, especially when control loops can compensate for drift. However, in a waveguide mesh, small imperfections in the couplers can leak light out of the main path. In an ASPIC, these losses are undesired, but they only result in a penalty in the transmission. In a waveguide mesh, this leaking light will propagate along parasitic paths, and can recombine coherently with the main path, giving rise to strong fluctuations. To compensate this, additional programming strategies are needed that not only control the state of the actuators along the main path, but also along possible parasitic paths [13], [14].

To enable this functionality for the users of the programmable PICs, a software development kit is needed that includes such configuration schemes, so one can develop high-level routines to use programmable PICs to explore new applications [2].

\section{SUMMARY}

Programmable photonic circuits can offer a new way of using photonic chips, similar to programmable electronics. The technology is enabled by high-density silicon photonics, but a programmable PIC platform is more than a photonic chip. It needs driver electronics and programming libraries to become a truly versatile tool for building new photonic functions.

\section{REFERENCES}

[1] X. Chen et al., "The Emergence of Silicon Photonics as a Flexible Technology Platform," Proceedings of the IEEE, vol. 106, no. 12, pp. 2101-2116, 2018.

[2] W. Bogaerts and A. Rahim, "Programmable Photonics: An Opportunity for an Accessible Large-Volume PIC Ecosystem," IEEE Journal of Selected Topics in Quantum Electronics2, vol. 26, no. 5, 20.

[3] J. Capmany and D. Perez, Programmable Integrated Photonics. Oxford University Press, 2020.

[4] D. Pérez et al., "Field-programmable photonic arrays," Optics Express, vol. 26, no. 21, p. 27265, 2018.

[5] M. Wang et al., "Tolerant, Broadband Tunable 2×2 Coupler Circuit," Optics Express, vol. 28, no. 4, pp. 5555-5566, 2020

[6] D. A. B. Miller, "Self-configuring universal linear optical component," Photonics Research, no. 1, pp. 1-15, 2013.

[7] N. C. Harris et al., "Linear programmable nanophotonic processors," Optica, vol. 5, no. 12, p. 1623, 2018.

[8] C. Errando-Herranz et al., "MEMS for Photonic Integrated Circuits," IEEE Journal of Selected Topics in Quantum Electronics, vol. 26, no. 2, pp. 1-1, 2019.

[9] A. Ribeiro et al., "Column-row addressing of thermo-optic phase shifters for controlling large silicon photonic circuits," Journal on Selected Topics in Quantum Electronics, vol. 15, pp. 1-8, 2020.

[10] M. Milanizadeh et al., "Control and calibration recipes for photonic integrated circuits," IEEE Journal of Selected Topics in Quantum Electronics, no. c, pp. $1-11,2020$.

[11] X. Chen et al., "Graph Representations for Programmable Photonic Circuits," Journal of Lightwave Technology, vol. 38, 2020.

[12] A. Lopez et al., "Auto-routing algorithm for field-programmable photonic gate arrays," Optics Express, vol. 28, no. 1, pp. 737-752, 2020.

[13] I. Zand and W. Bogaerts, "Effects of Coupling and Phase Imperfections in Programmable Photonic Hexagonal Waveguide Meshes," Photonics Research, vol. 8, no. 2, 2019.

[14] D. Pérez and J. Capmany, "Scalable analysis for arbitrary photonic integrated waveguide meshes," Optica, vol. 6, no. 1, p. $19,2019$. 